Mr Cartwright also gave some details of the damage caused to the calandria by mercury introduced either by accident or, possibly, by an industrial saboteur. The mercury had damaged the aluminium alloy vessel locally, he said, and a standpipe had had to be replaced, along with some other pipework. This entailed getting into the calandria, a difficult operation carried out by cutting a hole in the side. The calandria was now chemically clean, thanks partly to the use of nitric acid, Mr Cartwright said, and he was quite happy with the situation.

\section{Mass Formulae}

\section{from a Correspondent in High Energy Physics}

A RECENT article by Schwinger (Phys. Rev. Letters, 18,797 ; 1967) contains a mass formula for the two basic baryonic multiplets of the Eightfold Way theory of elementary particles which may throw some light on the question of how the theoretically simple group structure is violated in nature.

In the Eightfold Way theory, the basic baryon multiplet is an octet of particles with spin one-half comprising the nucleon $\mathrm{N}\left(939, \frac{1}{2}, 1\right)$ and the hyperons $\Sigma(1192,1,0), \Lambda(1115,0,0)$ and $\Xi(1318$, $\left.\frac{1}{2},-1\right)$. The numbers after the particle symbol are respectively the mass (in units of $\mathrm{MeV}$ ), the isotopic spin $I$, and the hypercharge $Y$. Each symbol denotes a group of particles making up an isotopic sub-multiplet and distinguished only by their charge. (Each group consists of $2 I+1$ particles of isotopic spin $I$.)

Their masses differ very little from the mean value quoted. If the SU(3) symmetry of the theory were exact, all particles of the octet would have the same mass, and it is hoped that by studying the way in which the masses of a given multiplet are split, some insight will be gained about the mechanism involved.

Simple group theoretic considerations led to the pro. posal of the now famous Gell-Mann-Okubo mass formula (S. Okubo, Prog. Theoret. Phys., 27, 949; 1962), which states that the mass of a member of a multiplet characterized by $I$ and $Y$ is given by

$$
m_{0}+m_{1} Y+m_{2}\left\{4 I(I+1)-Y^{2}\right\}
$$

where the three parameters $m_{0}, m_{1}$ and $m_{2}$ vary from multiplet to multiplet. It was verified that this form of the equation holds for the baryon octet deseribed and, subject to certain technical adjustments such as the use of squared masses and octet-singlet mixing angles, was found also to be applicable to the octets of pseudoscalar and vector mesons. For the case of the baryon decuplet which comprises thespin three-halves resonances $N^{*}(1238,3 / 2,1), \quad Y^{*}(1358,1,0), \Xi^{*}\left(1532, \frac{1}{2},-1\right)$ and $\Omega^{-}(1674,0,-2)$, the equality $Y=(I-1)$ holds and the formula yields equal spacing in $Y$. This can be seen to be quite reasonable, although the prediction of the mass of the $\Omega^{-}$particle was a memorable featthe particle had not then been found experimentally.

With the advent of the more restrictive $\mathrm{SU}(6)$ theory combining the concepts of isotopic spin (or unitary spin) and ordinary spin, Bég and Singh (Phys. Rev. Letters, 13, 418; 1964) showed that $m_{1}$ and $m_{2}$ should be the same for the baryon octet and decuplet. This result, which meant that the eight independent masses given here were to be fitted with only four parameters, was found to agree only moderately with experiment, thus confirming the view that the $\mathrm{SU}^{-}(6)$ symmetry is seriously violated, if it is valid at all.

Now Schwinger has observed that the masses can be fitted quite well with $m_{1}$ to $m_{2}$ in the ratio of -9 to 2. He effectively writes the mass formula for these multiplets as

$$
M_{0}+\Delta\left\{-3 Y / 2+\left[I(I+1) / 3-Y^{2} / 4+1\right]\right\}
$$

The ratio $\Delta / M_{0}$ is the same $(0 \cdot 119)$ for both octet and decuplet. Thus he has obtained a three parameter fit to the eight masses in which the fractional mass variation of approximately one-ninth is presumably a measure of the violation of the symmetry. It will be interesting to see how soon, if ever, this empirical exercise is followed by a physical interpretation.

\section{Animals in Laboratories from a Correspondent}

THe fifth annual meeting of the Society for Laboratory Animal Science (Gesellschaft für Versuchstierkunde), held earlier this month in Prague, was devoted this year to the nutrition and metabolism of laboratory animals. It covered a variety of species, from the usual rodents to the Japanese quail.

Dr Busse from Biberach (West Germany), who reported on recent work in rabbit nutrition, has found that these animals can live on vegetable food only and do not appear to be dependent at all on animal protein. He also confirmed earlier suggestions that rabbits appear to suckle their young only once in $24 \mathrm{~h}$, the suckling period lasting for $5 \mathrm{~min}$ and usually occurring in the early morning. Milk production by the doe can rise from $30 \mathrm{~g}$ at birth to $300 \mathrm{~g}$ prior to weaning. Because rabbits are very susceptible to intestinal infections between 40 and 60 days, the criterion for good breeding performance and high level of nutrition is the general appearance of the young at 70 days.

Failure of the glucose metabolism in clinically healthy dog bitches was described by Dr K. Gartner, Frankfurt/ Main. The dogs have an increased susceptibility to diabetes mellitus after oestrus. Deviation from the normal lasts from 60 to 80 days and the changes were repeated after subsequent oestrus. Ovariectomy during the corpora lutea stage had no effect, and further work suggests that increases in the secretion of insulin are followed by exhaustion in some cases. These symptoms are similar to the signs of latent diabetes mellitus in man.

The advantages of animals free from specified pathogens were further demonstrated by $\mathrm{Dr}$ W. H. Weire, Zurich. Fifty per cent of conventional male rats died by 710 days of age compared with only 10 per cent of their counterparts free from pathogens. These studics also underlined the need for providing particular diets for specific strains of animals maintained under constant conditions. The meeting proposed that standardized diets should be formulated in relation to internationally recognized strains of animals. A new criterion of "death rate" should also be introduced as well as fertility and weaning rate. Quality control of diets must be improved and there is the additional demand for "experimental diets" with strict adherence to composition. Professor Drepper (West Berlin) proposed that the use of synthetic additives (aminoacids, for example) would remove some of the variables 